\section{Why opening a door is as easy as eating an apple: $A$ reply to Thompson-Schill and Botvinick (2006)}

\author{
RANDI C. MARTIN and MICHAEL D. BYRNE \\ Rice University, Houston, Texas
}

Martin and Cheng (2006) present evidence suggesting that difficulty in verb generation is related to the strength of associative links between nouns and verbs and not to competition from alternative verb responses. Specifically, they show that latencies for verb generation are related to the associative strength of the most frequently produced verb and not to the ratio of the strength of the first to the second most frequently produced verb. Thompson-Schill and Botvinick (2006) have provided modeling results indicating how the findings of Martin and Cheng might be accommodated in a competitive model. Here we discuss how two noncompetitive models account for the findings and why such models should be preferred.

In the verb generation task, subjects are provided with a noun and must produce a semantically related verb. The verbs that subjects produce are typically actions that the noun performs (e.g., broom-sweep) or actions that a person or other animate entity performs on the noun (e.g., apple-eat). The task has been used frequently in neuroimaging studies of language processing, presumably because it is a semantic task that results in strong neural activations. It was used in the seminal PET study of word processing by Petersen, Fox, Posner, Mintun, \& Raichle (1989) and showed, rather surprisingly, neural activation in a left frontal region; this result was surprising because semantic representations have typically been associated with left temporal regions, on the basis of patient lesionfunction correlations (see, e.g., Mummery et al., 2000). In contrast to the flurry of subsequent imaging studies that have used the task, there have been few (if any) standard cognitive studies that have tried to pin down the cognitive operations involved in verb generation.

Martin and Cheng (2006; henceforth MC06) address one issue about the verb generation task - whether the degree of competition from alternative verbs determines the difficulty in providing a response. Previous findings from Thompson-Schill and colleagues had been interpreted as supporting a competition account (Thompson-Schill, D'Esposito, Aguirre, \& Farah, 1997; Thompson-Schill et al., 1998). However, there was a confound in the design of their studies, in that the ratio of the relative strength of the first to the second most frequent verb response (used

Preparation of the manuscript was supported by NIH Grant DC00218 to the first author. Correspondence relating to this article may be addressed to R. C. Martin, Psychology MS-25, Rice University, P.O. Box 1892, Houston, TX 77251 (e-mail: rmartin@rice.edu). to define degree of competition) was correlated with the strength of the most frequent response. When Martin and Cheng unconfounded these factors, they found that latencies for young and old healthy subjects and for a patient with left frontal damage were related to the strength of the most frequent verb response and not to the relative strength of that response to the nearest competitor. Thompson-Schill and Botvinick (2006; henceforth TSB06) have provided a response to MC06 in which they discuss how models that include competition could account for the MC06 findings. Here, we discuss how models without competition could account for these findings, as well as why such noncompetitive models might be preferred.

A simple noncompetitive model might be proposed in which one assumes a semantic network with associative links between noun concepts and related attributes, actions, category terms, and so forth. Activation of the noun concepts leads to a spread of activation throughout the network. In order for verbs to be given more weight, one might assume that the concept "action" is also activated by virtue of the task instructions and that the conjoint activation from the noun and from "action" determines the response (see, e.g., Anderson et al., 2004; see also our Figure 1). The strength of the spread of activation depends on the strength of the associative links between the noun and its associated concepts (and also between the "action" node and its association to verb concepts-but to simplify matters, we will assume these are equal). Activation of related verb concepts will build up over time, with the buildup dependent on the strength of the associative link. Once the activation of one of these concepts exceeds threshold, that verb is produced. Unlike the model presented in TSB06, in this case there are no lateral inhibitory links. Also, unlike other models of word selection (e.g., Roelofs, 2003), we do not assume that the criterion for selecting a verb depends on some ratio of the activation of the most activated node to others, since that would imply a role for competition. This simple model for the relation of associative strength to latency provides a straightforward account of the reaction time findings in MC06. That is, the association strength between the noun and the most frequently produced verb is directly reflected in the reaction time to produce the verb, irrespective of the strength of competing responses. If we assume that the role of the left frontal lobe is to maintain the activation of the specific noun concept and of the action concept, then the longer this maintenance is required on each trial, the greater the activation would be (Anderson et al., 2004).

One might instead produce a more elaborate model along the lines of that of Wagner, Paré-Blagoev, Clark, and Poldrack (2001), in which there are two different ways in which verbs are retrieved. The first is like the one described above, involving an automatic search through semantic memory through a spread of activation, with this automatic search dependent on processes carried out in 


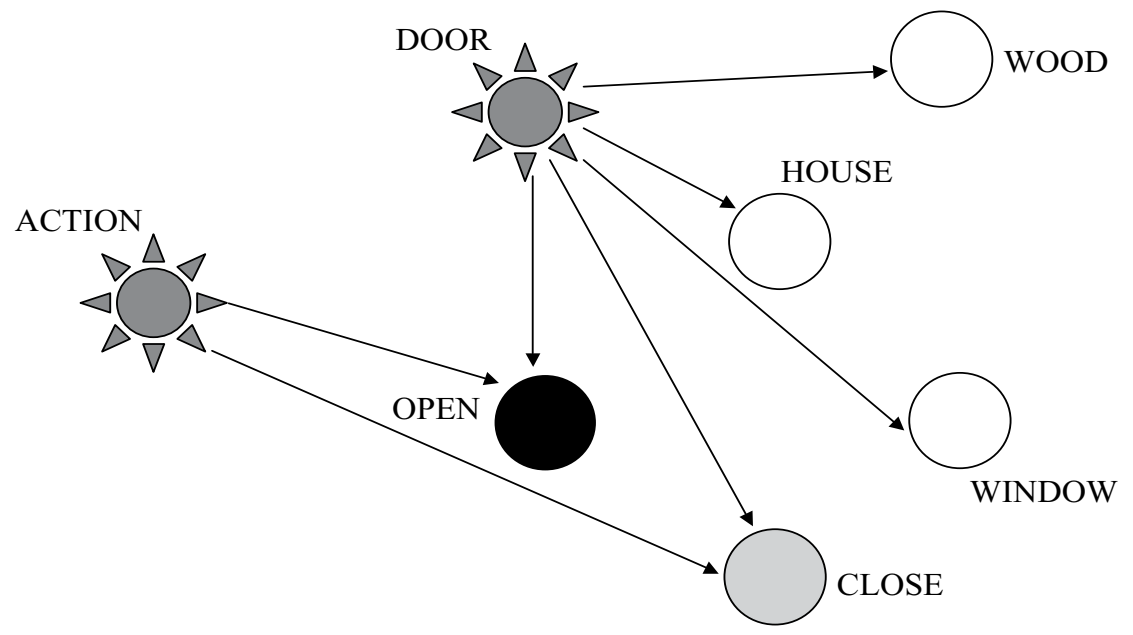

Figure 1. Noncompetitive model showing spreading activation from the concept associated with a noun stimulus (door) combined with that from an "action" concept. The darkness of the associated concept nodes indicates the degree of activation.

left temporal regions. One might assume, however, that if no response crosses threshold within a certain amount of time, a second mechanism kicks in, which involves a controlled search of semantic memory. As Wagner et al. argued, one could assume that the left frontal region is engaged only for this controlled search. How might a controlled search be carried out? The authors of TSB06 seem to imply that no meaningful distinction can be made between a controlled search and "selection." Consider the following possibility, however. One might assume that when no verb is forthcoming, the activation of the "action" concept is strategically lessened, such that a nonverb associate can become highly activated and cross threshold. In this case, the nonverb associate that has been retrieved and the "action" concept could be jointly activated in order to find a verb related to the nonverb associate through spreading activation. For example, for the noun chin there are no strong verb associates. Thus, if no verb comes to mind after some period of time, a reduction in the weight given to "action" might lead to a noun associate of chin, such as beard, becoming activated above threshold. Searching for joint associates of beard and "action" might lead to the retrieval of shave or scratch. The subject might, in such situations, carry out some check to make sure that the retrieved verb seems to have some relation to the original noun stimulus. It should be noted that the difficulty in coming up with a verb for a noun like chin is not in selecting one verb from a host of competitors, but in finding anything that seems reasonable. In this formulation, having a strong nonverb associate helps rather than hinders the eventual location of a response.

Either of these models (spreading activation alone or spreading activation plus controlled search) could account for the MC06 findings and provide an account of imaging and patient findings showing greater involvement of the left frontal region when there is no strong verb associate. In TSB06, the two findings that would seemingly cause difficulty for a competition explanation are accounted for by two different competitive factors. The first finding is that reaction times were substantially longer for nouns in the high-selection, low-association condition (e.g., rug) than in the high-selection, high-association condition (e.g., door), despite matching on the ratio of the strength of the association for the first and second most frequently produced verb associates. Thompson-Schill and Botvinick attribute this finding to a role for nonverb competitors. That is, even though the strength of the highest nonverb response was matched across conditions in MC06, the ratio of the highest nonverb response to the highest verb response would be higher when all verbs are weakly associated. Depending on the weight given to nonverb associates, these could provide competition that slowed down responding in the high-selection, low-association (rug) condition. This is a valid point that cannot be ruled out on the basis of the findings reported in MC06. One would predict, however, that reaction times would be slower, the greater the strength of nonverb competitors. An examination of the data from young subjects in MC06 for the high-selection, low-association condition revealed that the simple correlation of reaction time with strength of the highest nonverb response was -.03 and that the partial correlation was -.01 , with strength of the strongest verb associate partialed out. Thus, there is little suggestion that nonverb strength played an important role. Nonetheless, these calculations are based on the data from only 15 verbs, and thus the results should be treated with caution.

The second finding in MC06 that caused difficulty for a competition account was that reaction times did not differ for the low-selection (high association) condition (e.g., apple) and the high-selection, high-association condition (e.g., door), despite the fact that the nouns in the former condition had only one strongly associated verb (e.g., apple $\rightarrow$ eat), whereas nouns in the latter condition had two highly associated verbs (e.g., door $\rightarrow$ open, close). 
To model this finding, TSB06 assumed that resting-level strengths of the possible verb responses would vary randomly, so that the starting value would sometimes be higher for the second strongest verb. Then, if lateral inhibition between the two strongest verbs was strong, the higher initial activation of the weaker verb would slow down the activation of the stronger verb in the low-selection condition, although the imbalance in strengths of association would mean that the higher strength verb would eventually win out. (For the high-selection, high-association condition, either verb might win the competition, because they are closer in strength of association.) An examination of the distribution of reaction times could address these assumptions, since the TSB06 model predicts a bimodal distribution of reaction times in the low-selection condition for production of the highest associate, depending on whether that verb or the second strongest verb had a higher initial activation. In the simple model without competition, we predict long reaction times only when a nondominant response is produced. There are too few subjects and items in MC06 for a statistically meaningful test of the two models, particularly given that few subjects produced nondominant responses in the low-selection condition. Future research could certainly address this issue.

Although some modeling results are presented in TSB06, the authors indicate that they have not attempted to provide a model with a close fit to the reaction time data, but rather to provide a demonstration that parameters could be found for a competition-based model that could provide a qualitative account of the findings in MC06. We do not doubt that it is possible to find a set of parameter values that generates equivalent mean response times for the low-selection (apple) and high-selection, high-association (door) conditions - though it is not clear just how many parameters were explored to produce this result — but it should be noted that such a model does not address the effects of nonverb competitors; that would require a different explanation. It is not clear what network topology and parameter set would be required to accommodate both sets of results, or even whether such a combination exists at all. Nor is it clear whether such a combined model would have fewer free parameters than the number of data points being modeled. (The suggestion that the model is Bayesian in nature implies that at least the parameter estimate issue could be resolved by deriving parameters from conditional probabilities, but no such probabilities were presented.)

Even if a set of parameters could be found that might fit both findings adequately, one could still ask whether the more complicated model with the pair of factors put forward in TSB06 is preferable to the simple model shown in Figure 1. Without the collection of further data that might decide the issue, one might prefer the simpler model on the grounds of parsimony. Nonetheless, if one looks beyond the data in MC06 to other findings, a model that includes competition might have more support. As noted in MC06, many findings in the speech production literature have been taken to support a role for competition in word selec- tion (e.g., Schriefers, Meyer, \& Levelt, 1990). Recently, however, Miozzo and Caramazza (2003) and Finkbeiner and Caramazza (in press) have provided evidence against the competition account of these findings. Thus, the issue is far from settled.

In their comments on MC06, Thompson-Schill and Botvinick (2006) have concentrated on what they view as unresolvable issues in distinguishing between selection from competition and from controlled search. As discussed above, we are not particularly wedded to a dichotomy between automatic and controlled search, although such an approach may have some appeal on the basis of other findings. At the heart of what separates our views from those expressed in TSB06 is the role of competition in determining selection of a response. As discussed above, further empirical findings and additional, more precise modeling may be able to provide a more solid basis for deciding the issue. At present, a model assuming no competition seems to provide a simpler account of the findings.

\section{REFERENCES}

Anderson, J. R., Bothell, D., Byrne, M. D., Douglass, S., Lebiere, C., \& Qin, Y. (2004). An integrated theory of the mind. Psychological Review, 111, 1036-1060.

Finkbeiner, M., \& CARAMAZZA, A. (in press). Now you see it, now you don't: On turning semantic interference into facilitation in a Strooplike task. Cortex.

Martin, R. C., \& Cheng, Y. (2006). Selection demands versus association strength in the verb generation task. Psychonomic Bulletin \& Review, 13, 396-401.

Miozzo, M., \& Caramazza, A. (2003). When more is less: A counterintuitive effect of distractor frequency in the picture-word interference paradigm. Journal of Experimental Psychology: General, 132, 228-252.

Mummery, C. J., Patterson, K., Price, C. J., Ashburner, J., FrackOWIAK, R. S. J., \& HodGES, J. R. (2000). A voxel-based morphometry study of semantic dementia: Relationship between temporal lobe atrophy and semantic memory. Annals of Neurology, 47, 36-45.

Petersen, S. E., Fox, P. T., Posner, M. I., Mintun, M., \& Raichle, M. E. (1989). Positron emission tomographic studies of the processing of single words. Journal of Cognitive Neuroscience, 1, 153-170.

RoElofs, A. (2003). Goal-referenced selection of verbal action: Modeling attentional control in the Stroop task. Psychological Review, 110, $88-125$.

Schriefers, H., Meyer, A. S., \& Levelt, W. J. (1990). Exploring the time course of lexical access in language production: Picture-word interference studies. Journal of Memory \& Language, 29, 86-102.

Thompson-Schill, S. L., \& BotVINICK, M. M. (2006). Resolving conflict: A response to Martin and Cheng (2006). Psychonomic Bulletin \& Review, 13, 402-408.

Thompson-Schill, S. L., D'Esposito, M., Aguirre, G. K., \& Farah, M. J. (1997). Role of left inferior prefrontal cortex in retrieval of semantic knowledge: A reevaluation. Proceedings of the National Academy of Sciences, 94, 14792-14797.

Thompson-Schill, S. L., Swick, D., Farah, M. J., D’Esposito, M., KAN, I. P., \& KNIGHT, R. T. (1998). Verb generation in patients with focal frontal lesions: A neuropsychological test of neuroimaging findings. Proceedings of the National Academy of Sciences, 95, 15855 15860.

Wagner, A. D., Paré-Blagoev, E. J., Clark, J., \& Poldrack, R. A. (2001). Recovering meaning: Left prefrontal cortex guides controlled semantic retrieval. Neuron, 31, 329-338.

(Manuscript received May 6, 2006; revision accepted for publication May 9, 2006. ) 